\title{
Switchable nonlinear metalloferroelectric photonic crystals
}

\author{
E. Mishina, ${ }^{\text {a) }}$ A. Zaitsev, N. Ilyin, N. Sherstyuk, and A. Sigov \\ Moscow State Institute of Radioengineering, Electronics and Automation, Prospekt Vernadskogo 78, 119454 \\ Moscow, Russia \\ Yu. Golovko and V. Muhortov \\ South Center of Russian Academy of Science, 344006 Rostov-on-Don, Russia
}

\author{
A. Kolesnikov and Yu. Lozovik \\ Institute of Spectroscopy of Russian Academy of Sciences, 142190 Troitsk, Russia
}

M. Yemtsova and Th. Rasing

Institute for Molecules and Materials, Radboud University Nijmegen, Toernooiveld 1, 6525ED Nijmegen, The Netherlands

(Received 22 May 2007; accepted 29 June 2007; published online 25 July 2007)

\begin{abstract}
Two types of low-voltage electroswitchable nonlinear photonic crystals were fabricated providing spatial-frequency control of second harmonic generation radiation of visible light. A two-dimensional photonic crystal was fabricated by focused ion beam etching of a ferroelectric $\mathrm{BaSrTiO}_{3}$ thin film and switched by an electric field applied by an interdigital electrode system, which simultaneously acted as a one-dimensional photonic crystal. The fabricated device operated at a second harmonic wavelength around $400 \mathrm{~nm}$ with an efficiency incomparably higher than in the case of linear optics. (C) 2007 American Institute of Physics. [DOI: 10.1063/1.2762284]
\end{abstract}

Photonic crystals $(\mathrm{PhCs})$ are essential ingredient for the miniaturization of photonic components and the development of monolithic photonic integrated circuits. Typical integrated systems will include passive and active twodimensional (2D) photonic elements: waveguides, switches, multiplexers, and demultiplexers. Active elements imply tunability or switchability. Tuning of 2D photonic crystals is possible through the temperature dependence of the refractive index ${ }^{1}$ or the electro-optical effect. ${ }^{2}$

Although $\mathrm{LiNbO}_{3}$ ultrafast electro-optical modulaters were proposed ${ }^{3}$ recently, the advantage of $\mathrm{BaSrTiO}_{3}$ (BST) over $\mathrm{LiNbO}_{3}$ was demonstrated. ${ }^{4}$ Contrary to $\mathrm{LiNbO}_{3}$, BST is well compatible with planar technology and integration and possesses ultrafast low-voltage in-plane switching accompanied by very high nonlinear susceptibilities. This is why BST is very attractive to be used as a basis for switchable nonlinear photonic crystals.

In this letter we report the fabrication and demonstration of two types of nonlinear switchable photonic crystals based on thin BST ferroelectric films. These photonic crystals allow to control the direction of second harmonic generated (SHG) light propagation by applying low voltages to the system.

The $\mathrm{Ba}_{0.8} \mathrm{Sr}_{0.2} \mathrm{TiO}_{3}$ films of $80 \mathrm{~nm}$ thickness were fabricated by rf sputtering on a $\mathrm{MgO}$ substrate. Details on crystallographic and domain structure as well as switching properties were reported in Ref. 5. Copper interdigital planar electrodes with a period $d=2.2 \mu \mathrm{m}$ and a $800 \mathrm{~nm}$ gap width were patterned onto the BST film lithographically and simultaneously served as a one-dimensional metalloferroelectric photonic crystal $(\mathrm{PhC} 1)$. The size of the patterned area was $1 \times 0.2 \mathrm{~mm}^{2}$. The scanning electron microscopy (SEM) image of the structure is shown in Fig. 1(a).

2D square lattice photonic crystal structures of $50 \times 50$ and $100 \times 100 \mu \mathrm{m}^{2}$ were formed in the electrode-free areas

\footnotetext{
${ }^{a)}$ Electronic mail: mishina_elena57@mail.ru
}

in the ferroelectric films using focused ion beam milling (Quanta 200 3D, FEI Beam Technology). The SEM image of the obtained structure is shown in Fig. 1(b). The hole diameter was $300 \mathrm{~nm}$ with a period in the $X$ direction of $D_{2 \mathrm{D}}$ $=470 \mathrm{~nm}$. Since the holes were drilled only in the electrodefree areas (and not under the electrodes), there are two periods in the $Y$ direction: $470 \mathrm{~nm}$ and $1.89 \mu \mathrm{m}$; the former is equal to the period in the $X$ direction, and the latter is close to the period of the electrode structure.

In the gap between two electrodes two holes are located in the $Y$ direction. This superimposed structure, consisting of a two-dimensional $\mathrm{PhC}$ within the diffraction grating (or one dimensional $\mathrm{PhC}$ ), will be denoted in the following as $\mathrm{PhC} 2$. The depth of the holes was equal to the ferroelectric film thickness, which was controlled by the current change during the milling.

Voltages up to $\pm 8 \mathrm{~V}$ were applied to alternating electrodes, which provided oppositely directed electric fields up to $\pm E=10^{5} \mathrm{~V} / \mathrm{cm}$ between any pair of neighboring interdigital electrodes. Spatial modulation of the electric field results in modulation of (i) the refractive index via the electro-optic effect and (ii) the nonlinear optical susceptibility due to the in-plane ferroelectric polarization. Under electric field application the original electrode grating can be considered as

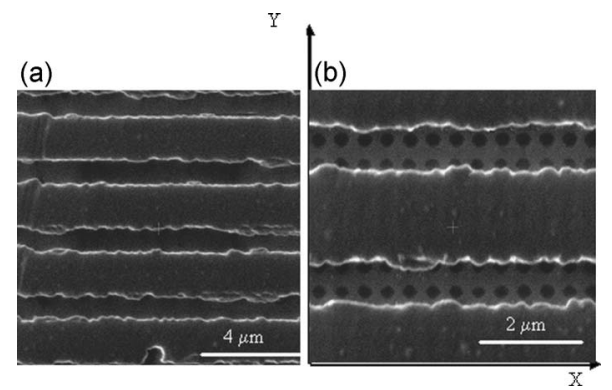

FIG. 1. Electron microscopic images of PhC1 (a) and $\mathrm{PhC} 2$ (b) metalloferroelectric photonic crystals. 


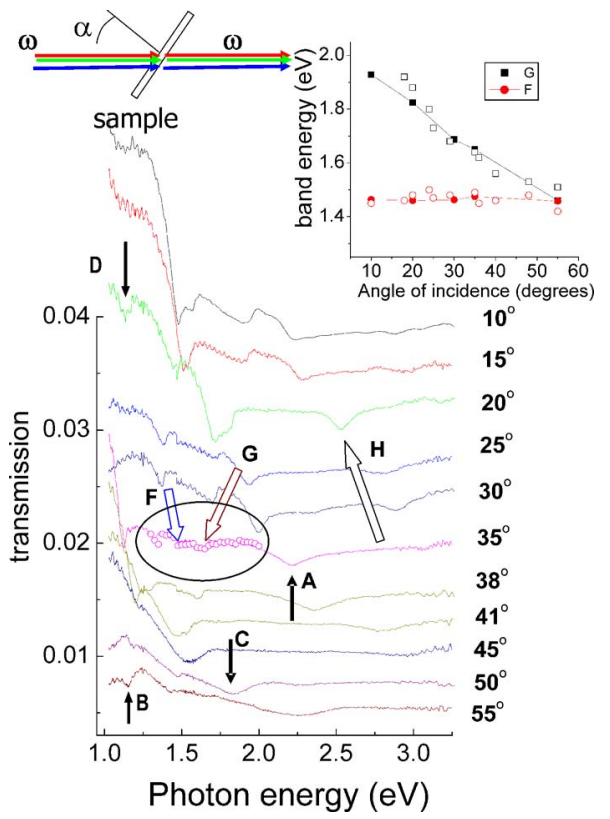

FIG. 2. (Color online) Transmission spectra for different angles of incidence (AOI) for $\mathrm{PhC} 2$, the corresponding $\mathrm{AOI}$ value is written near the spectral curves. The letters A-D denote the bands arising from one-dimensional $\mathrm{PhC}$; the letters $\mathrm{F}-\mathrm{H}$ and dashed area denote the bands arising from twodimensional PhC. Inside the ellipse by open circles-3D-FDTD calculated transmission for the angle of incidence of $35^{\circ}$. Inset right: dispersion curves of FG band of two-dimensional photonic crystal: experiment (solid circles) and 3D-FDTD modeling (open circles). Inset left: experimental geometry.

consisting of two subgratings with a doubled period and different refractive indices $n=n_{0} \pm \Delta n(E)$ and opposite nonlinear susceptibilities $\pm \Delta \chi(E)$.

Transmission spectra at different angles of incidence were measured by a variable angle spectroscopic ellipsometer in the range of $350-1100 \mathrm{~nm}(1.13-3.54 \mathrm{eV})$. The spectrally filtered radiation of the xenon light source was focused onto a spot of $200 \mu \mathrm{m}$ in diameter. Light was polarized with the electric field vector perpendicular to the metal strips (in the $Y Z$ plane).

For the SHG measurements the output of a Ti:sapphire laser was used in the range of $730-850 \mathrm{~nm}$ with a pulse width of about $100 \mathrm{fs}$, a repetition rate of $82 \mathrm{MHz}$, and an average power of $100 \mathrm{~mW}$ focused onto a spot of about $50 \mu \mathrm{m}$. A transmission geometry was used at normal incidence and the diffracted SHG light was measured by a photomultiplier tube with an aperture of about $1^{\circ}$ located on a rotating arm in a range of diffraction angles between $0^{\circ}$ and $50^{\circ}$. Both fundamental and SHG waves were polarized with the electric field vector perpendicular to the metal strips (in the $Y Z$ plane). The signal-to-noise ratio of the SHG measurements varied between 200 and 10, decreasing with the diffraction order.

Figure 2 shows transmission spectra of $\mathrm{PhC} 2$. For each angle of incidence, several minima in the transmission are observed denoted by a Latin letter. Minima from A to D exist not only in $\mathrm{PhC} 2$ but also in the transmission spectra of $\mathrm{PhC1}$ as well (not shown separately here). Minima from $\mathrm{F}$ to $\mathrm{H}$ appear for $\mathrm{PhC} 2$ only. Simulations of the transmission spectra were made with a three-dimensional (3D) finitedifference time-domain (FDTD) method $^{6}$ with subpixel smoothing for increased accuracy. For oblique incident plane waves we used a spectral FDTD technique described in Ref. 7. At the top and the bottom of the computational cell, perDownloaded 04 Jul 2008 to 131.174.20.208. Redistribution subject

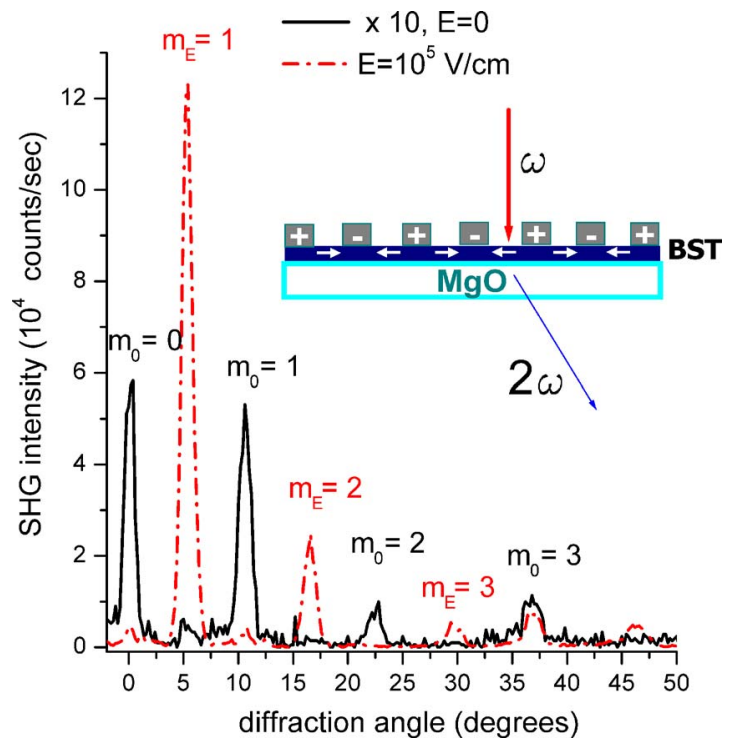

FIG. 3. (Color online) Nonlinear (SHG) diffraction on PhC1 without (multiplied by 10$)$ and with electric field application $(2.5 \mathrm{~V})$ for the SHG wavelength of $415 \mathrm{~nm}$. Inset: schematic side view of $\mathrm{PhCl}$; white arrows denote electric field direction between electrodes.

fectly matched layers were placed. For BST, an average value of the refractive index was taken between $\mathrm{BaTiO}_{3}$ and $\mathrm{SrTiO}_{3}{ }^{8}$

An example of the calculated transmission spectrum in the range of the $\mathrm{FG}$ band for $35^{\circ}$ angle of incidence is shown in Fig. 2 (marked by a circle). Corresponding dispersion curves (experimental and calculated) for the FG band (inset of Fig. 2) show excellent agreement. No difference in transmission was observed for both types of PhCs when an electric field was applied to the samples.

The minima in the transmission spectra appear due to energy transfer from the incident wave to the photonic crystal modes with excitation efficiency depending on geometry and materials. For 2D PhCs the photonic band gap (PBG) manifests itself as an attenuation dip in the transmission spectrum. ${ }^{9}$ The position and the width of the PBG depend on geometrical parameters and on the angle of incidence, while the contrast (or attenuation value) depends mostly on the efficiency of the coupling of the incident light with the photonic modes. ${ }^{10}$ Besides, for our sample the low attenuation values result from the high ratio of the light spot diameter relative to the $2 \mathrm{D} \mathrm{PhC}$ areas (about 50).

In contrast to the linear-optical behavior, the application of an electric field drastically changes the spatial distribution of second harmonic light. Figure 3 and 4 show diffraction patterns of the SHG light $I^{2 \omega}\left(\theta_{\text {diffr }}\right)$ for $\mathrm{PhCl}$ and $\mathrm{PhC} 2$, respectively.

In the diffraction patterns one can distinguish two types of diffraction maxima, which exist without and with electric field application and are denoted in the following as $m_{0}$ (zero field) and $m_{E}$ (electric field induced), respectively. Positions of $m$ maxima are determined by the metal grating period and fit the nonlinear Bragg law: ${ }^{11} \mathbf{k}_{2 \omega}=2 \mathbf{k}_{\omega}+\mathbf{Q}_{l}$, where $k_{\omega}$ and $k_{2 \omega}$ are wave vectors of the fundamental and SH waves and $\mathbf{Q}_{l}=2 \pi / d$ is the reciprocal grating vector. For normal incidence, positions of the main maxima can be found from the grating equation $\sin \theta_{0}^{2 \omega}=\left(\lambda_{2 \omega} / d\right) m_{0}\left(m_{0}=1,2, \ldots\right)$, which exactly coincide with the experimental data.

to AIP license or copyright; see http://apl.aip.org/apl/copyright.jsp 

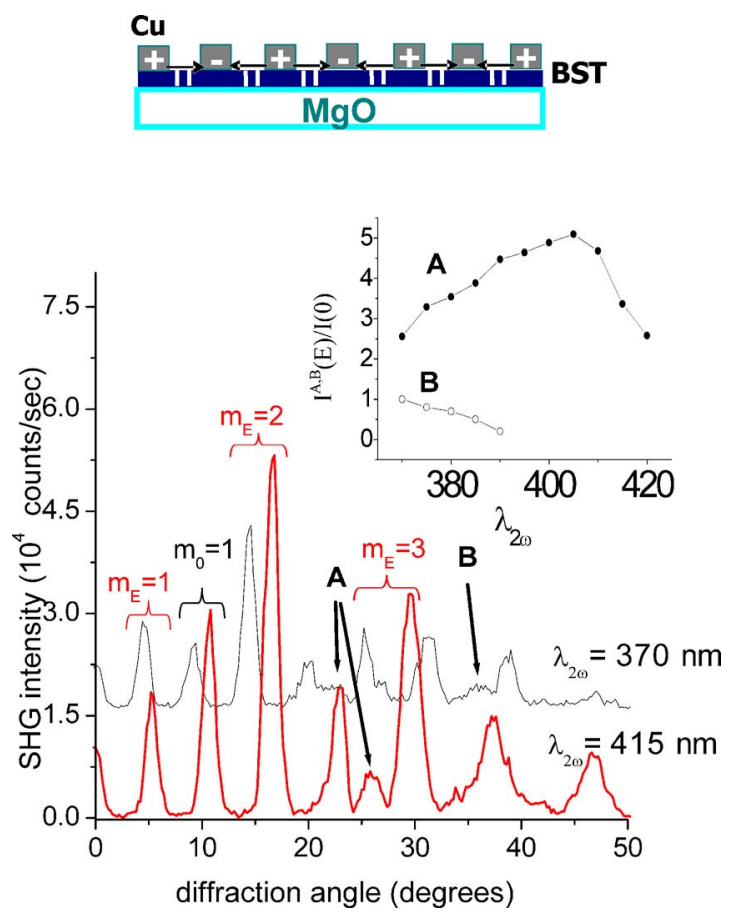

FIG. 4. (Color online) Nonlinear (SHG) diffraction on $\mathrm{PhC} 2$ with electric field application $(2.5 \mathrm{~V})$ for two different $\mathrm{SH}$ wavelengths. Inset top: schematic side view of $\mathrm{PhC2}$; arrows denote electric field direction between electrodes. Inset right: spectral dependences of A and B diffraction bands.

The source of SHG in an unbiased ferroelectric film is the nonlinear polarization which results from the uncompensated polarization. When an electric field is applied the polarization is enhanced and the period of the ferroelectric grating is doubled because of the opposite polarization direction of the BST material in neighboring gaps (see inset of Fig. 3). The polarization maxima can be found from the nonlinear Bragg law $\mathbf{k}_{2 \omega}=2 \mathbf{k}_{\omega}+\mathbf{Q}_{l} / 2$ and grating equation $\sin \theta_{E}^{2 \omega}$ $=\left(\lambda_{2 \omega} / 2 d\right) m_{E}\left(m_{E}=1,3,5, \ldots\right)$, which again exactly coincide with the experimental data.

For $\mathrm{PhC} 2$, all the maxima observed have the same positions as for $\mathrm{PhC1}$, although the SHG intensity distribution is different and the maxima are wider. The main distinction is, however, the appearance of additional maxima (denoted as A and B in Fig. 4) for a biased sample at angles which satisfy the diffraction equation $\sin \theta_{E 2 \mathrm{D}}^{2 \omega}=\left(\lambda_{2 \omega} / 4 D_{2 \mathrm{D}}\right) m_{E 2 \mathrm{D}}$ $\left(m_{E 2 \mathrm{D}}=2,3\right)$. These electric-field induced maxima appear due to the doubling of the period of the 2D structure under electric field application. With a period of $D_{2 \mathrm{D}}=470 \mathrm{~nm}$, the angular position of the first zero-field diffraction maximum falls out of the range of observations. The same holds for the fourth polarization diffraction maximum with $m_{E 2 \mathrm{D}}=4$. The absence of the maxima with $m_{E 2 \mathrm{D}}=1$ is not clear yet.

The amplitudes of the additional maxima $\mathrm{A}$ and $\mathrm{B}$ are spectral dependent. For $m_{E 2 \mathrm{D}}=3$ the maximum is observed around $\lambda_{2 \omega}=410 \mathrm{~nm}$ (curve A in the inset of Fig. 4). For $m_{E 2 \mathrm{D}}=2$ the maximum is observed at the edge of the observation range at $\lambda_{2 \omega}=370 \mathrm{~nm}$ (curve B in the inset of Fig. 4).

In $\mathrm{PhC} 1$ the SHG intensity of the electric field induced maxima are almost zero without electric field, because the remnant and nonswitchable polarization is very small in BST films. When an electric field is applied, the electric field induced maxima are much stronger than the zero-field maxima, and the zero-field maxima may even disappear (see $m_{0}=2$ in Fig. 3 for $415 \mathrm{~nm}$ ). In $\mathrm{PhC} 2$ the electric-field induced maxima exist even for zero field and increase up to ten times in comparison with their unbiased value.

The most exciting feature of these fabricated $\mathrm{PhCs}$ is that they combine efficient generation of SHG with spatialwavelength redistribution of the generated light: applying an electric field changes the propagation direction of the generated SHG light over several degrees, while the deflection angle depends on the wavelength. The switching efficiency can be calculated as

$$
E=\frac{I_{m_{E}}^{\mathrm{SHG}}}{I_{m_{0}=0}^{\mathrm{SHG}}(0)} .
$$

For $\mathrm{PhC} 1$ the highest efficiency value $E=30$ is achieved for $m_{E}=1$ at $\lambda_{2 \omega}=420 \mathrm{~nm}$ and $E=15$ at $\lambda_{2 \omega}=370 \mathrm{~nm}$. For $\mathrm{PhC} 2$ the highest efficiency value $E=10$ is achieved for $m_{E}=2$ at around $\lambda_{2 \omega}=410 \mathrm{~nm}$. This is much larger than in the case of linear optics, in which efficiencies of only a few percent were reported. For PhC2, the SHG intensity of the polarization maxima $\mathrm{A}$ and $\mathrm{B}$ equals zero in the absence of electric field. Using Eq. (1) one can calculate the switching efficiency for maximum A: $E_{\mathrm{A}}=5$ around $400 \mathrm{~nm}$ and for maximum $\mathrm{B} E_{\mathrm{B}}=1$ around $360 \mathrm{~nm}$.

It is important to note that the size of the device of $50 \mu \mathrm{m}$ was chosen to allow easy optical visualization. The high switching contrast allows us to decrease the size by more than a factor of 2 .

In conclusion, two types of ferroelectric thin film based switchable nonlinear photonic crystals were fabricated providing spatial-frequency control of light. An interdigital electrode system on top of a ferroelectric $\mathrm{BaSrTiO}_{3}$ film represents a one-dimensional (1D) photonic crystal, which provides in-plane switching of the ferroelectric polarization. A 2D photonic crystal was fabricated on top of the $1 \mathrm{D} \mathrm{PhC}$ by focused ion beam etching. The highest switching efficiency observed for the obtained PhCs at the second harmonic wavelength was about 30 .

Parts of this work were supported by the Russian Foundation for Basic Research (grant Nos. 06-02-17534, 06-0208152), the Ministry of Science and Education of Russia, and Stichting voor Fundamenteel Onderzoek der Materie.

${ }^{1}$ S. W. Leonard, J. P. Mondia, H. M. van Driel, O. Toader, S. John, K. Busch, A. Birner, U. Gösele, and V. Lehmann, Phys. Rev. B 61, R2389 (2000).

${ }^{2}$ H. Takeda and K. Yoshino, Phys. Rev. E 67, 056607 (2003).

${ }^{3}$ B. M. A. Rahman, S. Haxha, V. Haxha, and K. T. V. Grattan, Proc. SPIE 6389, 63890X (2006).

${ }^{4}$ P. Tang, D. J. Towner, T. Hamano, A. L. Meier, and B. W. Wessels, Opt. Express 12, 5962 (2004).

${ }^{5}$ E. D. Mishina, N. E. Sherstyuk, V. I. Stadnichuk, A. S. Sigov, V. M. Mukhorotov, Yu. I. Golovko, A. van Etteger, and Th. Rasing, Appl. Phys. Lett. 83, 2402 (2003).

${ }^{6}$ A. Taflove and S. C. Hagness, Computational Electrodynamics: The Finite-Difference Time-Domain Method (Artech, Norwood, MA, 2000), pp. 67-106.

${ }^{7}$ A. Aminian and Y. Rahmat-Samii, IEEE Trans. Antennas Propag. 54, 6 (2006).

${ }^{8}$ E. D. Palik, Handbook of Optical Constants of Solids (Academic, New York, 1988), Vol. 2, pp. 797 and 1042.

${ }^{9}$ A. Christ, S. G. Tikhodeev, N. A. Gippius, J. Kuhl, and H. Giessen, Phys. Rev. B 91, 183901 (2003).

${ }^{10}$ O. Parriauxy, V. A. Sychugov, and A. V. Tishchenko, Pure Appl. Opt. 5, 453 (1996).

${ }^{11}$ I. Freund, Phys. Rev. Lett. 21, 1404 (1968). 